(C) 2017 IEEE. Reprinted, with permission, from M. M. Habib, R. A. Welikala, A. Hoppe, C. G. Owen, A. R. Rudnicka and S. A. Barman, "Microaneurysm detection in retinal images using an ensemble classifier," 2016 Sixth International Conference on Image Processing Theory, Tools and Applications (IPTA), Oulu, 2016, pp. 1-6. doi: $10.1109 /$ IPTA.2016.7820998 


\title{
Microaneurysm Detection in Retinal Images Using an Ensemble Classifier
}

\author{
M.M. Habib ${ }^{1}$, R.A. Welikala ${ }^{1}$, A. Hoppe ${ }^{1}$, C.G. Owen ${ }^{2}$, A.R. Rudnicka ${ }^{2}$ and S.A. Barman ${ }^{1}$ \\ ${ }^{1}$ School of Computing, Faculty of Science, Engineering and Computing, Kingston University, London, United Kingdom \\ e-mail: m.habib@kingston.ac.uk, s.barman@kingston.ac.uk \\ 2 Population Health Research Institute, St. George's, University of London, United Kingdom
}

\begin{abstract}
Diabetic Retinopathy (DR) is one of the leading causes of blindness amongst the working age population. The presence of microaneurysms (MA) in retinal images is a pathognomonic sign of DR. In this work we have presented a novel combination of algorithms applied to a public dataset for automated detection of MA in colour fundus images of the retina. The proposed technique first detects an initial set of candidates using a Gaussian Matched filter and then classifies the initial set of candidates in order to reduce the number of false positives. A Random Forest ensemble classifier using a set of 79 features (the most common features used within literature) was used for classification. Our proposed algorithm was evaluated on a subset of 20 images from the MESSIDOR dataset. We show that the use of the Random Forest classifier with the 79 features improves the sensitivity of the detection, compared to using a K-Nearest Neighbours classifier that has been proposed in other techniques. In addition, the Random Forest is capable of ranking features according to their importance. We have ranked the 79 features according to their importance. This ranking provides an insight into the most important features that are necessary for discriminating true MA candidates from spurious objects. Eccentricity, aspect ratio and moments are found to be among the important features.
\end{abstract}

Keywords_-Image processing, Medical Image Analysis, Retinal Imaging, Microaneurysm Detection, Random Forest, Diabetic Retinopathy.

\section{INTRODUCTION}

Diabetic Retinopathy (DR) is one of the leading causes of blindness in the working age population, and over $2 \%$ of the population in the UK is affected [1]. Fundus images of the retina are used to diagnose DR. The appearance of microaneurysms (MA) is a pathognomonic sign of DR (Figure 1). Computer-aided detection and diagnosis of MA in retinal images has been an active area of research due to its application in DR detection and adaptability to public screening programs. However, MA detection is still a challenging problem due to the varying size and shape of MA in retinal images [2].

In general, most MA detection techniques have three main stages in common: 1) preprocessing, 2) MA Candidate detection and 3) candidate classification. The main purpose of preprocessing is to correct for non-uniform illumination. MA Candidate detection seeks to detect an initial set of regions where MA candidates are likely to exist. Ideally, the initial candidates detection phase should detect majority of the candidates and some false positive regions. A comparison of several candidate detection steps is presented in Murugan [3]. MA candidate classification uses a classifier to improve the sensitivity of the algorithm by filtering out false positives from the previous stage. Based on some features the classifier determines whether it is a true candidate or false positives. There are a few unsupervised techniques that do not rely on a classification stage [4][10].

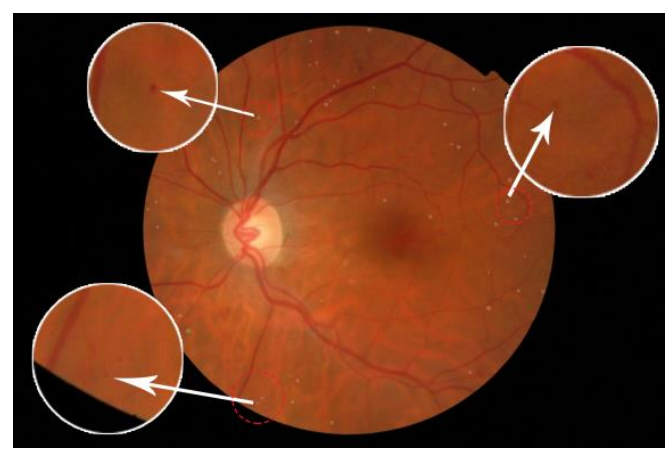

Figure 1. A colour retinal image with microaneurysms of various contrasts highlighted.

Early algorithms have performed MA detection on flourescene angiograms [11]-[13]. In these methods, a Gaussian matched filter was used to detect the initial set of microaneurysms and a rule-based classifier was used to classify the set to filter out false candidate detections. More recent techniques have tackled the problem of MA detection on colour fundus images. The main reason for this is that colour images, unlike flourescene images, are more common in screening programs and are also non-invasive to capture.

The methods explained in this paragraph are all based on MA detection in colour fundus images. A large portion of the methods rely on a Gaussian matched filter [14]-[16], or a variant of the Gaussian filter [7], [17], [18] in order to detect the initial set of candidates. Other methods for initial candidate detection include thresholding [8], [9], [19], Moat operator [20], double ring filter [21], mixture modelbased clustering [10] 1D scan lines [4], [5], extended minima transform [22], [23], Hessian matrix Eigenvalues [24], [25], Frangibased filters [26] and hit-or-miss transform [27]. A variety of classification techniques have been used in order to reduce the number of false positive detections. These include Linear Descriminant Analysis (LDA) [14] K-Nearest Neighbours (KNN) [15], [16], [18], [25], Artificial Neural Networks [21], [27], Naive Bayes [22] and Logistic Regression [28]. A number of techniques did not rely on a classifier (unsupervised methods) [4], [5], [8], [9] . These techniques have the advantage of eliminating the need for a training set, but may not perform as well as supervised methods.

Haloi [29] recently applied deep neural networks to detect MAs in colour images. Deep neural networks have gained popularity in the field of computer vision in the recent years since they do not require manual feature engineering (selection of features). Moreover, algorithms based on deep learning have produced results that outperform other state-of-the-art algorithms in other computer vision applications. However, deep learning requires massive datasets for training [30] and such large labeled retinal image datasets are not commonly available. 
The objective of the present work is as follows: 1) to present a new technique for microaneurysm detection based on an ensemble classifier for classification. 2) Introduce 79 of the most common features used in the literature and perform feature ranking in order to identify the features that are most important for discriminating microaneurysm candidates from spurious objects.

The rest of the paper proceeds as follows. Section II describes the methodology of the proposed algorithm. In Section III, the experiments that were performed to assess the algorithm are described, results presented and discussed. A final discussion and concluding remarks are presented in Section IV.

\section{METHODOLOGY}

The proposed algorithm is based on the technique proposed by Fleming [16] with two main variations in the classification stage 1) an extended set of 79 features has been used and 2) A Random Forest classifier was used rather than K-Nearest Neighbours (KNN). The algorithm consists of three main stages A) Image preprocessing, B) Initial Candidates Detection and C) Candidates classification. In the following subsections we describe the stages involved in the algorithm while highlighting the main contributions that the technique introduces.

\section{A. Preprocessing}

The preprocessing steps proceed as follows: Given a colour image input, $I_{\mathrm{RGB}}$, the green channel $I_{G}$ is extracted since MA candidates have the highest contrast in this channel (Figure 2(a)). $I_{\text {med }}$, a median filtered image is generated by applying a $3 \times 3$ median filter to $I_{G}$ for the purpose of salt \& pepper noise removal. $I_{\text {adapt }}$ is generated by performing Contrast-adaptive histogram equalization to $I_{\text {med }}$ in order to enhance the contrast in the image (Figure 2(c)). $I_{\text {noise }}$ is generated by convolving a $3 \times 3$ Gaussian filter $(\sigma=2)$ with $I_{\text {adapt }}$ for further noise reduction. $I_{\text {shade }}$, a shade corrected image is generated in order to correct for non-uniform illumination, shade correction is applied to the contrast-enhanced image. During shade correction the background is estimated by applying a $68 \times 68$ median filter. The filter size is chosen to be large enough in order to eliminate vessels and other features in the image (producing $I_{b g}$ as shown in Figure 2(b)):

$$
I_{\text {shade }}=I_{\text {adapt }} / I_{\text {bg }}
$$

Global contrast normalization is performed on the resulting image by dividing it by its standard deviation. The normalized image $I_{\text {con }}$ is generated by:

$$
I_{\text {con }}=\frac{I_{\text {shade }}}{s t d\left(I_{\text {shade }}\right)}
$$

where $\operatorname{std}(x)$ represents the standard deviation of image x. Finally, blood vessels are removed from the image by applying a linear morphological black top-hat transform (also known as bottom-hat transform) in 8 directions $\left(\theta \in\left\{\theta_{i}: \theta_{i}=22.5 \times i ; i=[0 . .7]\right\}\right)$. The length of the tophat structuring element was chosen to be $15 \mathrm{px}$ long (measured as the width of the largest blood vessel in the images). The resulting image of the aforementioned operations is the preprocessed image $I_{\mathrm{pp}}$ (Figure 2(d)). Figure 2 shows examples of each preprocessing step mentioned in this section.

\section{A. MA Candidates Detection}

During this step we produce an initial set of candidates. The target is to maximize the amount of true candidates detected. The false positives will be reduced during the classification phase, therefore the highest priority in this phase is to maximize the amount of true positives [19].

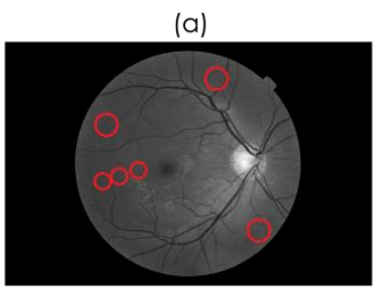

(c)

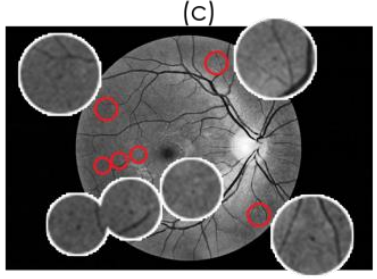

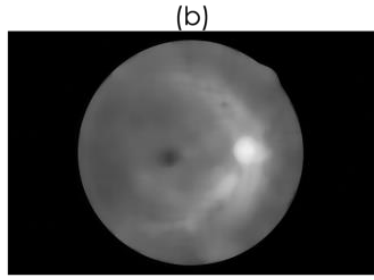

(d)

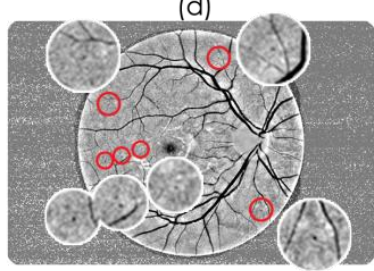

Figure 2. Proposed algorithm steps a) Green channel image, $I_{G}$ b) Estimated background $\left.\left(\boldsymbol{I}_{\boldsymbol{b} g}\right), \mathrm{c}\right)$ Histogram equalized image $\left(\boldsymbol{I}_{\text {adapt }}\right)$,

d) shade-corrected image ( $\left.\boldsymbol{I}_{\text {shade }}\right)$. In all images the labeled MA groundtruths have been overlayed in red.

A $15 \times 15$ Gaussian filter is applied to detect local minima regions in the image. The result of the Gaussian filter is a probability map between 0 and 1 where higher values indicate higher chance of that pixel being an MA pixel. The resulting image is $I_{\text {gauss }}$ :

$$
I_{\text {gauss }}=I_{p p} * \operatorname{gauss}(\sigma), \quad \text { where } \sigma=1.0
$$

The value of $\sigma$ was empirically chosen after trying a range of values. In order to get the initial candidates a thresholding operation is performed. The resulting image is $I_{\text {thresh }}$ :

$$
\mathrm{I}_{\text {thresh }}=\operatorname{thresh}\left(\mathrm{I}_{\text {gauss }}, \tau\right)
$$

Where thresh(f,t) is the thresholding operation applied to image $f$ using a threshold value of $t$. The value of $\tau$ is chosen such that at most $5 \%$ of the pixels will belong to the MA candidate regions [16].

In order to enhance the shapes of the detected microaneurysm candidates, a region growing operation was performed using the initial candidates as input. This region growing uses the same method documented in [16]. It involves iteratively growing along the connected components from the minimum intensity pixel until a maxima point is reached with respect to an energy function. The energy function is defined as the average value of the gradients around the boundary of the grown region. All the parameters of this stage have been kept the same except the maximum grown size. While the method suggested a maximum grown size of 3000 pixels, we found that this causes large blood vessel regions to be picked up as a result of this. We empirically found that a value of 100 pixels for the maximum area caused the amount of false positives to decrease significantly while achieving almost the same sensitivity. The value was chosen to be over twice the size of the average MA size in the groundtruth images. The result of this stage is the image $\mathrm{I}_{\mathrm{RG}}$.

\section{MA Candidates Classification}

The objective of the classification phase is to classify each MA candidate detected during the Candidates Detection phase as either an MA or a spurious object. The idea is to eliminate as many false 
positive candidates as possible (while retaining the true candidates). A classifier is used for this purpose. The classifier needs to be trained on some labeled example cases of both true and spurious candidates. Based on these examples it builds a model that is able to filter unseen examples as either true or spurious. The input to the classifier is a set of 'features' extracted from each candidate. These features need to be distinctive in order to allow the building of an accurate model.

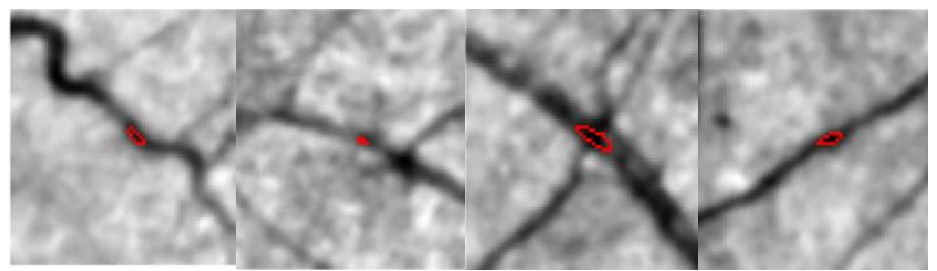

Figure 3. Examples of candidates falsely detected on vessels. Red circles represent intial candidate MA detected by the proposed method

For our proposed technique we have chosen to use Random Forests as our classifier. Random Forests is an ensemble-based technique based on decision-tree learning. An ensemble classifier is one which combines the decision of multiple weak classifiers. The reasons for our choice of this classifier are: 1) It has performed well for other computer vision tasks [31], [32]; 2) It can rank features as it builds the classification model, which is very insightful to know which of our features are most effective and 3) Decision-tree learners are robust to outliers and poor features [33]. We have extended Fleming's [16] feature set of 10 features to include a set of 79 features. These were based on the features that have been reported in the literature. Table 2 displays a list of the 79 features that were fed into the classifier. These features are explained below in the same order of appearance as the table:

- $\quad$ Fleming's features (1-9): These are the features introduced by Fleming in his technique [16]. Most of these techniques rely on fitting a paraboloid to each candidate's intensity profile in order to estimate some parameters from the paraboloid. These features are based on both the shape and intensity of the object. Details about these features is presented in the original paper [16].

- Vessel features: $(\mathbf{1 7}, \mathbf{2 5}, \mathbf{2 6})$ : These are binary features $(0$ or 1) that indicate whether or not a candidate seems to lie on a vessel. Since MA objects never lie on vessels [16], it is highly likely that a candidate that lies on a vessel is a spurious one [16] (Figure 3). Discriminating between vessels and MA candidates is quite important since we experimentally found that $70 \%$ of the initial candidates detected are false positives that lie on vessels [13]. The first isVessel feature (17) uses the method suggested by Fleming. However we found that this feature is not discriminative enough and does not detect many candidates that lie on vessels. Therefore we introduced two other features: isvessel_loose (25) and isvessel_QUARTZ (26). isvessel_loose is an adaptation of Fleming's isVessel feature with more relaxed constraints. isvessel_QUARTZ detects the vessel tree structure using [34] and marks any candidate that overlaps with this vessel structure as a spurious candidate.

- $\quad$ Shape features (10-16, 18-24): Moment Invariants (10-16) are 7 features that describe the shape of an object [35]. In order to calculate moments we crop a small region (101x101 px) centered around the candidate binary image and use that subimage to compute the 7 features for each candidate. Other shape features include aspect ratio, major \& minor axis length. Some of these feature overlap with Fleming's features, however these are calculated at a pixel level rather than after fitting a paraboloid to the candidate.
- Gaussian Features (27-58): Using the Gaussian matched filter response as features have been used extensively in literature. Since microaneurysms vary in size, the features have taken this into account by varying the value of $\sigma$ while applying the Gaussian filter (i.e. applying the filter at multiple scales). Some definitions related to these features will follow. Some of the symbols mentioned below are utilized in Table 2. Let $I_{\text {shade }}$ be the shade corrected image (Section II B) and:

$$
G_{\sigma}=I_{\text {shade }} * \operatorname{gauss}(\sigma)
$$

be the Gaussian filter response for sigma $=\sigma$ and $G_{\sigma}(x, y)$ be the filter response at coordinates $(x, y)$. Let $\sigma$ be the set of sigma values $\sigma \in\{1,2,4,8,16,32\}$. Let $\bar{C}$ be a set of initial candidates detected (after region growing). Each candidate (c) is a set of coordinates $\left(x_{i}, y_{i}\right)$. Let seed $(c)$ be the coordinates $\left(x_{s}, y_{s}\right)$ of the minimum intensity defined as follows:

$$
\begin{aligned}
& \operatorname{seed}(c)=\left(x_{s}, y_{s}\right) \\
& =\underset{(x, y) \in c}{\operatorname{argmin}}\left(I_{\text {shade }}(x, y)\right)
\end{aligned}
$$

A 1-Dimensional Gaussian is a special case of $G_{\sigma}$ applied linearly in one direction. $G_{s, t}^{1 D}(x, y)$ is the 1D Gaussian applied at angle $t$ and a scale (standard deviation) of $s$. In our case we have applied the 1D Gaussian at a constant scale $(s=1)$. Let the set $\theta$ be the set of angles applied at each coordinate. In our experiments:

$$
\theta \in\left\{\theta_{i}: \theta_{i}=10 * i ; \quad i=[0 . .9]\right\}
$$

- Intensity Features (59-76): These are calculated directly from the intensity in the image at multiple bands: the red $(\mathrm{R})$, blue (B), green $(\mathrm{G})$ band in the RGB colour space; the Hue $(\mathrm{H})$, saturation $(\mathrm{S})$ and value $(\mathrm{V})$ bands of the HSV space.

- Morphological Features (77-79): These three features are based on applying a linear morphological close operator (15 px) at different angles and are aimed at discriminating vessels from microaneurysms. This is because the linear structures of vessels would respond differently at different angles of the linear operator while the circular nature of MA objects would cause the response to be more uniform.

In the following section we describe the method that was used to assess the performance of the proposed algorithm.

\section{EXPERIMENTAL RESULTS}

In order to evaluate the performance of the proposed algorithm we have relied on a subset of the MESSIDOR dataset [36] . A set of 20 images were chosen from this dataset to cover a wide range of retinopathy as shown in Table 1 . The images were all either healthy or suffered from early-stage Diabetic Retinopathy (DR) and were good resolution images. There were no abnormalities such as laser scars in the images. The purpose of this selection was to have a dataset of ideal scenario images for assessment. This means that the results of the assessment on this dataset should produce the ideal performance of a given algorithm or technique. In other words, the dataset should identify the 'peak performance' of the algorithm being tested.

The images were groundtruthed by an expert grader. During the groundtruthing the grader marked all the microaneurysms that were visible to him. A circular marker was used rather than pixel-based 
marker [26]. Majority of the literature has relied on object-based metrics to measure the accuracy of detection. This is because it gives a more sensible measure of performance - indicating the amount of MA objects detected in the image relative to the total MA objects present. Furthermore, reliance on pixel-based metrics can be misleading due to the inbalance in proportion between very few MA pixels and a large number of background pixels.

The images in the dataset belonged to 1 of 3 different resolutions. In order to maintain uniformity, all the images were resized to the width of the smallest image (1440px) while maintaining the image aspect ratio. This was also done to speed up the processing of the images. During resizing 'bicubic interpolation' with antialiasing was used.

The dataset was split into 10 images for training and 10 images for testing. The training features were used to generate the model while the test features were used to measure the accuracy of the model.

One parameter that needs to be selected for the Random Forest classifier is the number of trees generated as a part of the model $\left(N_{\text {trees }}\right)$. A larger number of trees $\left(N_{\text {trees }}\right)$ reduces the error but it comes at the cost of increased computation. An advantage of the bagging process employed by Random Forests is that the generalization error can be estimated during the training process (outof-bag error). This makes it easier to select a suitable value for $N_{\text {trees }}$. In order to select a suitable value for $N_{\text {trees }}$ we perform the classification while varying this parameter and calculate the error for each step. The result of this is shown in Figure 4. Based on this process a value of $N_{\text {trees }}=150$ was selected since no significant error change at grown trees number $>150$

Table 1. Distribution of DR grades (a) and resolutions (b) of images in the dataset.

(a)

\begin{tabular}{|l|l|l|}
\hline $\begin{array}{l}\text { Retinopathy } \\
\text { Grade }\end{array}$ & $\begin{array}{l}\text { Number of } \\
\text { MAs }\end{array}$ & $\begin{array}{l}\text { Image } \\
\text { Count }\end{array}$ \\
\hline DR0 & 0 & 4 \\
\hline DR1 & $1-5$ & 7 \\
\hline DR2 & $6-14$ & 6 \\
\hline DR3 & $>15$ & 3 \\
\hline & TOTAL & 20 \\
\hline
\end{tabular}

In order to measure the accuracy of the model, we measured the sensitivity of the proposed method. Given image $I_{i}$ in our dataset (for $i=[1 . .20]$ ), let $G_{i}$ be the set of true MA objects (groundtruth) for image $I_{i}$ and $C_{i}$ be the set of detected candidates after classification (Section II C) for image $I_{i}$. The sensitivity is defined as:

$$
\text { Sensitivity }=\frac{\sum_{i=1}^{20}\left|G_{i} \cap C_{i}\right|}{\sum_{i=1}^{20}\left|G_{i}\right|}
$$

Where $|\ldots|$ represents set cardinality. Thus the sensitivity is the proportion of true candidates detected in proportion to the total number of true candidates. A candidate $\mathrm{c} \in \mathrm{C}$ is considered to be equivalent to $g \in G$ if the pixel coordinates of $g$ and $c$ overlap by at least 1 pixel. Note that we are measuring the sensitivity on a candidate level rather than on a pixel level. Since we cannot determine the number of true negatives, we used a Free Receiver Operating Curve (FROC) rather than a traditional ROC curve [11]. In an FROC curve, the $\mathrm{x}$-axis is replaced with the average number of false positive candidates per image instead of the specificity. Figure 5 shows the FROC curve for both Random Forest (black) and K-Nearest Neighbours (red). The blue curve represents the performance of Fleming's state-of-the-art algorithm on our dataset, the red curve represents the performance of Fleming's algorithm with the extended feature set (Table 2). A value of $K=15$ was used for the $\mathrm{KNN}$ classifier [16]. In order to generate the FROC curves, the 'strictness' of each classifier was varied. In the case of the KNN classifiers used by Fleming, the k-threshold value was varied between 1 and 15 [16]. The Random Forest classifier produces a probability value (P) between 0 and 1 representing the likelihood of a candidate of belonging to 0 or 1 . We use a threshold value $\mathrm{P}_{\mathrm{t}}$ to produce the final classification (i.e. class $=0$ if $\mathrm{P} \leq \mathrm{P}_{\mathrm{t}}$, otherwise class $=1$ ). In the case of the Random Forest classifier we varied the value of $P_{t}$ to generate the FROC curve. Random Forests generate trees at random and generates the attribute splits at random as well [31], [32]. Due to this feature of the classifier, every run produces results with slightly different accuracy. To overcome the varying results, we have applied the Random Forest classifier multiple times and presented the best (solid line), worst (dotted line) and median (dashed line) performance result as shown in Figure 5. Based on the figure we note that including additional features to the KNN classifier used by Fleming improves the performance (as shown by the blue and red curves). Furthermore, we can see that an ensemble classifier outperforms the KNN classifier used by Fleming (as shown by the blue and red curves), suggesting that Random Forests are more robust to outlier features.

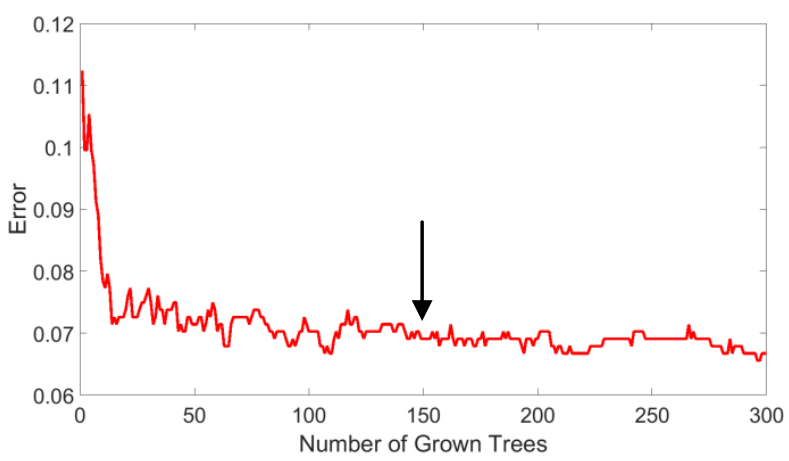

Figure 4. Out-of-bag (OOB) Classification Error Vs Number of trees in Random Forest classifier.

One of the interesting advantages of the Random Forest Classifiers is that it can measure the importance of each feature. This gives us an indication of the features that have the most discriminative power to separate a true candidate from a spurious one. Figure 6 shows the feature importance for each of the 79 features. Feature importance is computed using the average mean squared error (MSE) at each node. Among the important features are some shape features such as eccentricity, 2nd moment and aspect ratio; some features that discriminate MAs from vessels such as morphological tophat; intensity features such as the mean candidate intensity (red channel), mean candidate intensity (Hue channel), candidate contrast [21] (red channel), candidate standard deviation (shade corrected image), and the Standard deviation of the Gaussian matched filter response $(\sigma=$ 1 ). This suggests that a diverse set of feature types is necessary in order to discriminate true microaneurysms from spurious ones. Interestingly, the binary features isVessel, isVessel_loose and isVessel_QUARTZ were not among the effective features.

\section{SUMMARY AND CONCLUSIONS}

This work introduces a new approach based on Fleming's method for micornauerysm detection that relies on a Random Forest ensemble classifier (bagging) for microaneurysm classification. Our evaluations on a set of 20 images from the MESSIDOR dataset showed that the use of the Random Forest as a classifier improves the performance over the use of a K-Nearest Neighbors (KNN) classifier used by Fleming. This is mainly because the Random Forest classifier is less affected by noise or outlier features (due to the way decision tree learners work) [33]. In addition, Random Forests are also very 
efficient to train and can be easily parallelized across multiple computers or threads, since each tree is independent of the other trees. We have ranked the importance of 79 common features that have appeared in the literature and concluded that a diverse range of features are important to distinguish between true microaneurysms and spurious candidates.

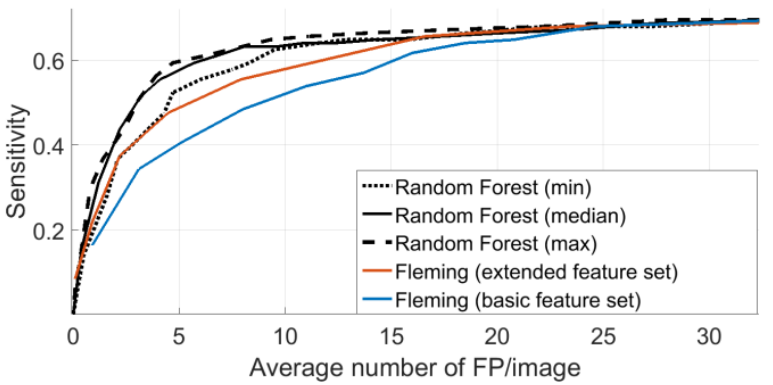

Figure 5. FROC curve of the proposed algorithm compared to Fleming [17]

Table 2. Features list. Some symbols below are defined in Section III.

\begin{tabular}{|c|c|c|c|}
\hline Index & Feature name & Index & Feature name \\
\hline 1 & Number of peaks & $36-41$ & $G_{\sigma_{i} \in \sigma}(\operatorname{seed}(c))$ \\
\hline 2 & Major Axis length & $42-47$ & $\operatorname{mean}_{(x, y) \in c}\left(G_{1}(x, y)\right)$ \\
\hline 3 & $\begin{array}{l}\text { Mean of minor and major } \\
\text { axis }\end{array}$ & $48-53$ & $\operatorname{std}_{(x, y) \in c}\left(G_{\sigma_{i}}(x, y)\right)$ \\
\hline 4 & Eccentricity & 54 & $G_{2}(\operatorname{seed}(c))-G_{1}(\operatorname{seed}(c))$ \\
\hline 5 & Depth of candidate & 55 & $G_{4}(\operatorname{seed}(c))-G_{2}(\operatorname{seed}(c))$ \\
\hline 6 & Depth of candidate & 56 & $G_{8}(\operatorname{seed}(c))-G_{4}(\operatorname{seed}(c))$ \\
\hline 7 & Energy & 57 & $G_{16}(\operatorname{seed}(c))-G_{8}(\operatorname{seed}(c))$ \\
\hline 8 & $\begin{array}{l}\text { candidate depth / mean } \\
\text { diameter of MA candidate }\end{array}$ & 58 & $G_{32}(\operatorname{seed}(c))-G_{16}(\operatorname{seed}(c))$ \\
\hline 9 & Energy with depth correction & 59 & Sum of candidate intensities (R) \\
\hline $10-16$ & Moment Invariants & 60 & Sum of candidate intensities (G) \\
\hline 17 & isVessel & 61 & Sum of candidate intensities (B) \\
\hline 18 & $\begin{array}{l}\text { Aspect Ratio (major axis } \\
\text { length / minor axis length) }\end{array}$ & 62 & $\begin{array}{l}\text { Sum of candidate intensities } \\
\text { (shade) }\end{array}$ \\
\hline 19 & major axis length & 63 & mean candidate intensity $(\mathrm{R})$ \\
\hline 20 & minor axis length & 64 & mean candidate intensity $(\mathrm{G})$ \\
\hline 21 & Perimeter & 65 & mean candidate intensity (B) \\
\hline 22 & Area & 66 & mean candidate intensity (shade) \\
\hline 23 & Eccentricity & 67 & $\begin{array}{l}\text { standard deviation of the candidate } \\
\text { (shade) }\end{array}$ \\
\hline 24 & Compactness & 68 & Max - min candidate value $(\mathrm{R})$ \\
\hline 25 & isVessel (loose) & 69 & max - min candidate value $(\mathrm{G})$ \\
\hline 26 & isVessel (QUARTZ) & 70 & max - min candidate value $(B)$ \\
\hline 27 & $\max _{s \in \sigma}\left(G_{s}(\operatorname{seed}(c))\right)$ & 71 & candidate contrast $(\mathrm{R})$ \\
\hline 28 & $\min _{s \in \sigma}\left(G_{s}(\operatorname{seed}(c))\right)$ & 72 & candidate contrast $(\mathrm{G})$ \\
\hline 29 & $\operatorname{mean}_{s \in \sigma}\left(G_{s}(\operatorname{seed}(c))\right)$ & 73 & candidate contrast (B) \\
\hline 30 & $\max _{t \in \theta}\left(G_{1, t}^{1 D}(x, y)\right)$ & 74 & candidate contrast $(\mathrm{H})$ \\
\hline 31 & $\min _{t \in \theta}\left(G_{1, t}^{1 D}(x, y)\right)$ & 75 & candidate contrast (S) \\
\hline 32 & $\operatorname{mean}_{t \in \theta}\left(G_{1, t}^{1 D}(x, y)\right)$ & 76 & candidate contrast $(\mathrm{V})$ \\
\hline 33 & $\operatorname{std}_{t \in \theta}\left(G_{1, t}^{1 D}(x, y)\right)$ & 77 & $\begin{array}{l}\text { maximum candidate response of } \\
\text { the morph close ratio }\end{array}$ \\
\hline 34 & $\begin{array}{l}\text { 1D gaussian response at angle } \\
\text { perpendicular to the } \\
\text { maximum response (30) }\end{array}$ & 78 & $\begin{array}{l}\text { minimum candidate response of } \\
\text { the morph close ratio }\end{array}$ \\
\hline 35 & $\max (30,34)$ & 79 & $\begin{array}{l}\text { mean candidate response of the } \\
\text { morph close ratio }\end{array}$ \\
\hline
\end{tabular}

Among the important -features are those that discriminate the circular shape of the microaneurysm such as eccentricity, aspect ratio and moments. Features that are capable of discriminating MA candidates from vessels are also very important, since we have empirically found that $70 \%$ of the candidates that were spurious lied on vessels or on vessel cross sections. In other words, a classifier that manages to filter out false positives that lie on vessels will boost the sensitivity values greatly since $70 \%$ of the false positives will be eliminated. Morphological closing operator, and 1D gaussians are examples of features respond highly to microaneurysms and not very high to spurious objects of vessels.

To the best of our knowledge, this account on feature importance performed using a large feature set (79 features) has not been performed before and is therefore essential to present an insight for future work regarding the most important features that can discriminate microaneurysms to filter out spurious detections. A more extensive analysis of the 79 features, including feature selection, will be presented in future work.

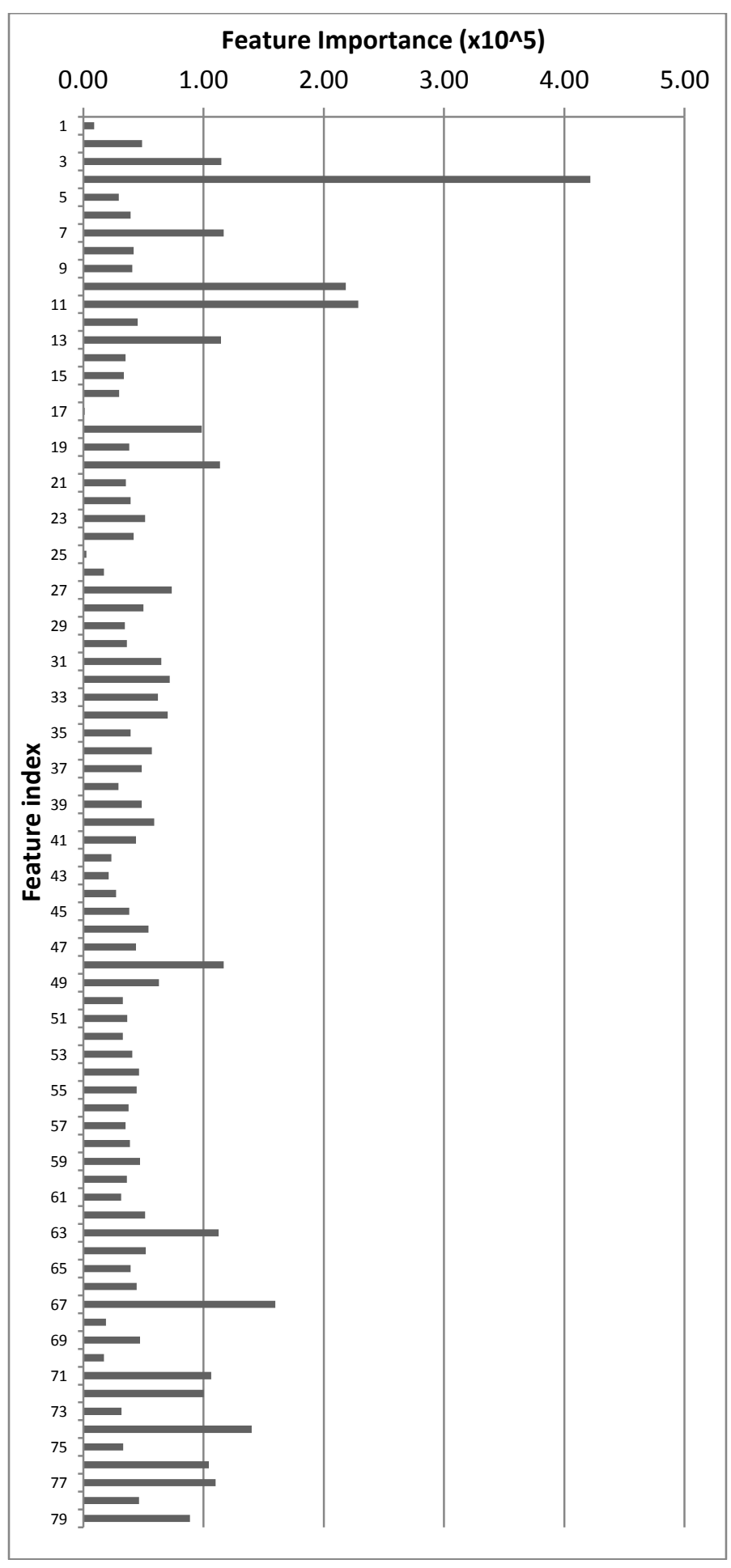

Figure 6. Feature importance as defined in Table 2. 


\section{REFERENCES}

[1] J. Evans, C. Rooney, F. Ashwood, N. Dattani, and R. Wormald, "Blindness and partial sight in England and Wales: April 1990-March 1991," Health Trends, vol. 28, no. 1, pp. 5-12, 1996.

[2] A. J. Frame, P. E. Undrill, M. J. Cree, J. A. Olson, K. C. McHardy, P. F. Sharp, and J. V. Forrester, "A comparison of computer based classification methods applied to the detection of microaneurysms in ophthalmic fluorescein angiograms," Comput. Biol. Med., vol. 28, no. 3, pp. 225-238, 1998.

[3] R. Murugan, R. Korah, S. Nasreen Fathima, and T. Venkata Haritha, "Microaneurysms detection methods in retinal images using mathematical morphology," Ophthalmology, vol. 110, pp. 1679-80, 2003.

[4] I. Lazar and A. Hajdu, "Microaneurysm detection in retinal images using a rotating cross-section based model," in 2011 IEEE International Symposium on Biomedical Imaging: From Nano to Macro, 2011, pp. 1405-1409.

[5] I. Lazar and A. Hajdu, "Retinal Microaneurysm Detection Through Local Rotating Cross-Section Profile Analysis," IEEE Trans. Med. Imaging, vol. 32, no. 2, pp. 400-407, Feb. 2013.

[6] S. B. Júnior and D. Welfer, "Automatic Detection of Microaneurysms and Hemorrhages in Color Eye Fundus Images," Int. J. Comput. Sci. Inf. Technol., vol. 5, no. 5, pp. 21-37, Oct. 2013.

[7] Q. Li, R. Lu, S. Miao, and J. You, "Detection of microaneurysms in color retinal images using multi-orientation sum of matched filter," in Proc. of the 3rd International Conference on Multimedia Technology, 2013.

[8] L. Giancardo, F. Mériaudeau, T. P. Karnowski, K. W. Tobin, Y. Li, and E. Chaum, "Microaneurysms detection with the radon cliff operator in retinal fundus images," 2010, p. 76230U.

[9] L. Giancardo, F. Meriaudeau, T. P. Karnowski, Y. Li, K. W. Tobin Jr, and E. Chaum, "Microaneurysm detection with radon transform-based classification on retina images," in Engineering in Medicine and Biology Society, EMBC, 2011 Annual International Conference of the IEEE, 2011, pp. 5939-5942.

[10] C. I. Sánchez, R. Hornero, A. Mayo, and M. García, "Mixture modelbased clustering and logistic regression for automatic detection of microaneurysms in retinal images," 2009, p. 72601M.

[11] T. Spencer, J. A. Olson, K. C. McHardy, P. F. Sharp, and J. V. Forrester, "An Image-Processing Strategy for the Segmentation and Quantification of Microaneurysms in Fluorescein Angiograms of the Ocular Fundus," Comput. Biomed. Res., vol. 29, no. 4, pp. 284-302, Aug. 1996.

[12] M. J. Cree, J. A. Olson, K. C. McHardy, P. F. Sharp, and J. V. Forrester, "A fully automated comparative microaneurysm digital detection system," Eye, vol. 11, no. 5, pp. 622-628, Sep. 1997.

[13] S. Abdelazeem, "Micro-aneurysm detection using vessels removal and circular hough transform," in Radio Science Conference, 2002.(NRSC 2002). Proceedings of the Nineteenth National, 2002, pp. 421-426.

[14] L. Streeter and M. J. Cree, "Microaneurysm detection in colour fundus images," Image Vis. Comput N. Z., pp. 280-284, 2003

[15] M. Niemeijer, B. van Ginneken, J. Staal, M. S. A. Suttorp-Schulten, and M. D. Abramoff, "Automatic detection of red lesions in digital color fundus photographs," IEEE Trans. Med. Imaging, vol. 24, no. 5, pp. 584-592, May 2005.

[16] A. D. Fleming, S. Philip, K. A. Goatman, J. A. Olson, and P. F. Sharp, "Automated microaneurysm detection using local contrast normalization and local vessel detection," IEEE Trans. Med. Imaging, vol. 25, no. 9, pp. 1223-1232, Sep. 2006.

[17] B. Zhang, X. Wu, J. You, Q. Li, and F. Karray, "Detection of microaneurysms using multi-scale correlation coefficients," Pattern Recognit., vol. 43, no. 6, pp. 2237-2248, Jun. 2010.

[18] J. Wu, J. Xin, L. Hong, J. You, and N. Zheng, "New hierarchical approach for microaneurysms detection with matched filter and machine learning," in Engineering in Medicine and Biology Society (EMBC), 2015 37th Annual International Conference of the IEEE, 2015, pp. 4322-4325.

[19] J. H. Hipwell, F. Strachan, J. A. Olson, K. C. McHardy, P. F. Sharp, and J. V. Forrester, "Automated detection of microaneurysms in digita red-free photographs: a diabetic retinopathy screening tool," Diabet. Med., vol. 17, no. 8, pp. 588-594, 2000.

[20] C. Sinthanayothin, J. F. Boyce, T. H. Williamson, H. L. Cook, E. Mensah, S. Lal, and D. Usher, "Automated detection of diabetic retinopathy on digital fundus images," Diabet. Med., vol. 19, no. 2, pp 105-112, Feb. 2002.

[21] A. Mizutani, C. Muramatsu, Y. Hatanaka, S. Suemori, T. Hara, and H Fujita, "Automated microaneurysm detection method based on double ring filter in retinal fundus images," 2009, p. $72601 \mathrm{~N}$.

[22] A. Sopharak, B. Uyyanonvara, and S. Barman, "Simple hybrid method for fine microaneurysm detection from non-dilated diabetic retinopathy retinal images," Comput. Med. Imaging Graph., vol. 37, no. 5-6, pp. 394-402, Jul. 2013.

[23] A. Sopharak, B. Uyyanonvara, and S. Barman, "Automatic microaneurysm detection from non-dilated diabetic retinopathy retinal images using mathematical morphology methods," IAENG Int. J. Comput. Sci., vol. 38, no. 3, pp. 295-301, 2011.

[24] T. Inoue, Y. Hatanaka, S. Okumura, C. Muramatsu, and H. Fujita, "Automated microaneurysm detection method based on eigenvalue analysis using hessian matrix in retinal fundus images," in Engineering in Medicine and Biology Society (EMBC), 2013 35th Annual International Conference of the IEEE, 2013, pp. 5873-5876.

[25] K. M. Adal, D. Sidibé, S. Ali, E. Chaum, T. P. Karnowski, and F. Mériaudeau, "Automated detection of microaneurysms using scaleadapted blob analysis and semi-supervised learning," Comput. Methods Programs Biomed., vol. 114, no. 1, pp. 1-10, Apr. 2014.

[26] R. Srivastava, D. W. Wong, L. Duan, J. Liu, and T. Y. Wong, "Red lesion detection in retinal fundus images using Frangi-based filters," in Engineering in Medicine and Biology Society (EMBC), 2015 37th Annual International Conference of the IEEE, 2015, pp. 5663-5666.

[27] R. Rosas-Romero, J. Martínez-Carballido, J. Hernández-Capistrán, and L. J. Uribe-Valencia, "A method to assist in the diagnosis of early diabetic retinopathy: Image processing applied to detection of microaneurysms in fundus images," Comput. Med. Imaging Graph., vol. 44, pp. 41-53, Sep. 2015.

[28] M. García, M. I. López, D. Álvarez, and R. Hornero, “Assessment of four neural network based classifiers to automatically detect red lesions in retinal images," Med. Eng. Phys., vol. 32, no. 10, pp. 1085-1093, Dec. 2010.

[29] M. Haloi, "Improved Microaneurysm Detection using Deep Neural Networks," ArXiv Prepr. ArXiv150504424, 2015.

[30] A. Krizhevsky, I. Sutskever, and G. E. Hinton, "ImageNet Classification with Deep Convolutional Neural Networks," in Advances in Neural Information Processing Systems 25, F. Pereira, C. J. C. Burges, L. Bottou, and K. Q. Weinberger, Eds. Curran Associates, Inc., 2012, pp. 1097-1105.

[31] O. CREDIT, "Bootstrap-inspired techniques in computational intelligence," IEEE Signal Process. Mag., vol. 1053, no. 5888/07, 2007.

[32] R. Polikar, "Ensemble based systems in decision making," IEEE Circuits Syst. Mag., vol. 6, no. 3, pp. 21-45, Third 2006.

[33] T. M. Mitchell, MACHINE LEARNING, International edition edition. New York: McGraw-Hill Education, 1997.

[34] "QUARTZ: Quantitative Analysis of Retinal Vessel Topology and size - An automated system for quantification of retinal vessels morphology." [Online]. Available:

http://www.sciencedirect.com/science/article/pii/S0957417415003504. [Accessed: 05-Jun-2016].

[35] M.-K. Hu, "Visual pattern recognition by moment invariants," IRE Trans. Inf. Theory, vol. 8, no. 2, pp. 179-187, Feb. 1962.

[36] E. Decencière, X. Zhang, G. Cazuguel, B. Lay, B. Cochener, C. Trone, P. Gain, R. Ordonez, P. Massin, A. Erginay, B. Charton, and J.-C. Klein, "FEEDBACK ON A PUBLICLY DISTRIBUTED IMAGE DATABASE: THE MESSIDOR DATABASE," Image Anal. Stereol., vol. 33, no. 3, pp. 231-234, Aug. 2014. 